Check for updates

Cite this: RSC Adv., 2017, 7, 33591

Received 14th May 2017

Accepted 20th June 2017

DOI: $10.1039 / c 7 r a 05443 b$

rsc.li/rsc-advances

\section{A ternary composite with manganese dioxide nanorods and graphene nanoribbons embedded in a polyaniline matrix for high-performance supercapacitors $\dagger$}

\author{
Tong Wu, ${ }^{a}$ Chaonan Wang, ${ }^{\mathrm{b}}$ Yao Mo, ${ }^{\mathrm{a}}$ Xinran Wang, ${ }^{\mathrm{a}}$ Jinchen Fan, (DD *a Qunjie $\mathrm{Xu}^{\star a}$ \\ and Yulin Min*a
}

A new ternary composite of manganese dioxide $\left(\mathrm{MnO}_{2}\right)$, polyaniline (PANI) and graphene nanoribbons (GNRs) has been fabricated through a two-step polymerization process. With manganese dioxide nanorods and graphene nanoribbons embedded in the PANI matrix, the $\mathrm{MnO}_{2} / \mathrm{GNRs} / \mathrm{PANI}$ ternary composites with 3-dimensional porous structures exhibited higher specific capacitance and better charge-discharge cycling performance than that of $\mathrm{MnO}_{2}$ and $\mathrm{MnO}_{2} / \mathrm{GNRs}$ binary composites in supercapacitors. The specific capacitance of the $\mathrm{MnO}_{2} / \mathrm{PANI} / \mathrm{GNRs}$ ternary composite can be achieved at $472 \mathrm{~F} \mathrm{~g}^{-1}$. After 5000 cycles, the specific capacitance with $79.7 \%$ of the initial capacitance can be preserved. The enhanced specific capacitance and cycle life implies the structural stability of $\mathrm{MnO}_{2} /$ GNRs/PANI ternary composites and the synergistic effect between $\mathrm{MnO}_{2}$, GNRs and PANI.

\section{Introduction}

Supercapacitors, also called ultracapacitors, have attracted considerable attention, owing to their high power density, longcycle performance, optimal operational safety and rapid charge-discharge rates. ${ }^{\mathbf{1}}$ In general, supercapacitors can be classified into two types depending on the charge storage mechanism and the corresponding active materials were used. Electrical double-layer capacitances (EDLCs), which store charges by reversible ion absorption between the electrode and electrolyte interface, mostly use carbon materials with high accessible surface area., ${ }^{2,3}$ Conventional carbon materials such as activated carbon, carbon nanotubes and carbon fiber usually exhibit good stability due to their special structures and large surface area. $^{4-7}$ Nevertheless, their specific capacitance is limited due to the low theoretical capacity derived from EDLCs. ${ }^{8-10}$ The other type, known as pseudocapacitors, is based on redox reactions that occur largely on material surfaces to store charges. ${ }^{\mathbf{1 1} 12}$ Typical pseudocapacitive materials include transition metal oxides, such as $\mathrm{RuO}_{2}, \mathrm{MnO}_{2}, \mathrm{NiO},{ }^{13,14} \mathrm{MoS}_{2}$ (ref.

\footnotetext{
${ }^{a}$ Shanghai Key Laboratory of Materials Protection and Advanced Materials in Electric Power, College of Environmental and Chemical Engineering, Shanghai University of Electric Power, Shanghai 200090, People's Republic of China.E-mail: Jinchen.fan@ shiep.edu.cn; xuqunjie@shiep.edu.cn; minyulin@shiep.edu.cn; Fax: +86 21 35303544; Tel: +862135303544

${ }^{b}$ College of Materials Science and Engineering, Guizhou Minzu University, Guiyang 550025, People's Republic of China
}

$\dagger$ Electronic supplementary information (ESI) available. See DOI: 10.1039/c7ra05443b
15) and conducting polymers like polyaniline (PANI), polypyrrole, and polythiophene. ${ }^{16-18}$ However, they usually display poor cycle performance with structural deformation during the charge and discharge processes. ${ }^{19}$ Undoubtedly, the capacitive performance of supercapacitors depend strongly on the performance of the electrode materials including their structures, surface area, conductivity and electrochemical stability. ${ }^{20}$ Therefore, increasing interest has been focused on the development of novel composites to enhance its capacitive performance. $^{21}$

Graphene nanoribbons (GNRs), thin elongated strips of graphene with a high length-to-width ratio and straight edges, can be successfully synthesized via longitudinal unzipping of MWCNTs by a chemical oxidation route. In recent years, GNRsbased materials have been extensively studied due to the high available surface area, extraordinary mechanical strength and outstanding electrical conductivity. ${ }^{22-24}$ The excellent electronic properties make them attractive especially as supporting materials for the fabrication of composites, and as so far, it has been studied in a wide range of applications such as batteries, supercapacitors and fuel cells. ${ }^{25-28}$

Nowadays, great interests are shown by researches on pseudocapacitance materials with excellent electrochemical characteristics such as metal oxides and conducting polymers. Among the metal oxides electrode materials, $\mathrm{MnO}_{2}$ has been studied extensively because of its advantages of low cost, natural abundance, environmental safety and high theoretical specific capacitance. However, the capacitance of $\mathrm{MnO}_{2}$ is limited by its poor electrical conductivity, which hinders the 
practical application in supercapacitors. On the other hand, among different kind of conducting polymers, PANI have been widely studied due to their controllable electrical conductivity and environmental stability. However, the drawbacks of the PANI electrode is also obvious, that is, a serious problem of typical volumetric swelling and shrinking happens during the charge-discharge process, which severely influence the cycle performance, limiting its practical usage in supercapacitors.

In recent years, binary or ternary composites of carbon materials, conducting polymers and metal oxides have been investigated to make full use of their advantages and overcome their disadvantages of each component. For example, multiwalled carbon nanotube/PANI/ $\mathrm{MnO}_{2}$ ternary coaxial nanostructures, ${ }^{29}$ graphene $/ \mathrm{MnO}_{2} / \mathrm{PANI}$ ternary composite, ${ }^{30}$ manganese ferrite/graphene/PANI nanostructure ${ }^{31}$ have been prepared to further enhance its capacitive performance. These researches have shown that it is an effective way by immobilizing carbon materials with pseudocapacitive conducting polymers and metal oxides. Because of the unique synergistic effects between carbon materials and pseudocapacitance materials may greatly improve the electrochemical properties of the hybrid composite.

In this paper, we report unique $\mathrm{MnO}_{2} / \mathrm{GNRs} / \mathrm{PANI}$ ternary composites with GNRs and $\mathrm{MnO}_{2}$ nanorods embedded in PANI matrix. The introduction of PANI can not only prohibit the stacking of GNRs, but also fastens all of them directly interconnected with $\mathrm{MnO}_{2}$ nanorods to form ideal conductive threedimensional networks. Therefore, the electron transfers between GNRs and $\mathrm{MnO}_{2}$ has been improved due to the electrical conductivity of PANI. On the other hand, GNRs provide mechanical structures which significantly reduce the volumetric change during the charge-discharge process. The $\mathrm{MnO}_{2} /$ GNRs/PANI ternary composites exhibit outstanding capacitive performance and excellent cycle performance with a highest specific capacitance of $472 \mathrm{~F} \mathrm{~g}^{-1}$ at a current density of $1 \mathrm{~A} \mathrm{~g}^{-1}$ and the capacitive retention of as high as $85 \%$ observed after 1000 cycles at a current density of $10 \mathrm{~A} \mathrm{~g}^{-1}$.

\section{Experimental}

\subsection{Materials}

MWCNTs (purity, >95\%; length, $\sim 10 \mu \mathrm{m}$; diameter, $>30 \mathrm{~nm}$ ) were purchased from Chengdu Organic Chemistry Co., Ltd (China). Potassium permanganate $\left(\mathrm{KMnO}_{4}\right)$, manganese(II) chloride tetrahydrate $\left(\mathrm{MnCl}_{2} \cdot 4 \mathrm{H}_{2} \mathrm{O}\right)$, ammonium persulfate $\left(\left(\mathrm{NH}_{4}\right)_{2} \mathrm{~S}_{2} \mathrm{O}_{8}\right)$, aniline, concentrated sulfuric acid $\left(\mathrm{H}_{2} \mathrm{SO}_{4}\right)$, and hydrogen peroxide ( $30 \%$ water solution, $\mathrm{H}_{2} \mathrm{O}_{2}$ ) were purchased from Shanghai Aladdin Bio-chem Technology Co., Ltd. All chemicals were used as received without further purification. All solutions were prepared with deionized (DI) water.

\subsection{Preparation of $\mathrm{MnO}_{2}$ nanorods ${ }^{32}$}

In a typical procedure, $0.32 \mathrm{~g}$ of $\mathrm{KMnO}_{4}$ and $0.6 \mathrm{~g}$ of $\mathrm{MnCl}_{2}$ $\cdot 4 \mathrm{H}_{2} \mathrm{O}$ were added to $40 \mathrm{~mL}$ of deionized water with vigorously stirring for $30 \mathrm{~min}$ until formed the homogeneous solution. And then, the precursor mixture solution was further transferred into a $50 \mathrm{~mL}$ of Teflon-lined autoclave and subsequently heated at $160{ }^{\circ} \mathrm{C}$ for $12 \mathrm{~h}$. Then, it was cooled to room temperature naturally. The precipitates were obtained by centrifugation and sequentially washing with deionized water for several times, and then dried in vacuum at $80{ }^{\circ} \mathrm{C}$ for $6 \mathrm{~h}$. Finally, the browncolored powder of the $\mathrm{MnO}_{2}$ nanorods was obtained.

\subsection{Preparation of GNRs}

GNRs were synthesized according to the method described by Tour et al. ${ }^{22}$ Briefly, $150 \mathrm{mg}$ of MWCNTs was first dispersed in $200 \mathrm{~mL}$ of $\mathrm{H}_{2} \mathrm{SO}_{4}$ by stirring for $1 \mathrm{~h}$. Then, $2.0 \mathrm{~g}$ of $\mathrm{KMnO}_{4}$ was then slowly added into the MWCNTs $/ \mathrm{H}_{2} \mathrm{SO}_{4}$ mixture under a bath of mixture of ice water. Afterward, the mixture was stirred at room temperature for $1 \mathrm{~h}$ and then heated to $60{ }^{\circ} \mathrm{C}$ for $1 \mathrm{~h}$. After that, the reacted products were pour into $1000 \mathrm{~mL}$ of deionized water, and then adding $10 \mathrm{~mL}$ of $\mathrm{H}_{2} \mathrm{O}_{2}$ under ice water bath. The products of GNRs were harvested by centrifugation and washed with $10 \%$ (v/v) hydrochloric acid and deionized water for several times, and finally dried at $50{ }^{\circ} \mathrm{C}$.

\subsection{Preparation of $\mathrm{MnO}_{2} / \mathrm{PANI} / \mathrm{GNRs}$ ternary composites}

The $\mathrm{MnO}_{2} / \mathrm{PANI} / \mathrm{GNRs}$ ternary composites were prepared by two-step in situ polymerization. $0.1 \mathrm{~g}$ of as-prepared GNRs were first added into $100 \mathrm{~mL}$ of water and sonicated for $1 \mathrm{~h}$ to form a yellow-brown GNRs/water dispersion. By then, $0.12 \mathrm{~g}$ of aniline was slowly added into the GNRs/water dispersion under vigorously stirred to obtain the stable GNRs/aniline suspension. After, $10 \mathrm{~mL}$ of mixture solution of hydrochloric acid and APS was slowly added into GNRs/aniline dispersion with magnetically stirring. The molar ratio of aniline, hydrochloric acid and APS was $1: 1: 1$. When the reaction was conducted in ice bath for 10 minutes, $0.6 \mathrm{~g}$ of $\mathrm{MnO}_{2}$ nanorods dispersed in $50 \mathrm{~mL}$ of $1 \mathrm{M} \mathrm{HCl}$ water solution was added to the above reacting dispersion. Almost simultaneously, $0.12 \mathrm{~g}$ of aniline were added again and stirred for $4 \mathrm{~h}$. Finally, the deep-green $\mathrm{MnO}_{2} / \mathrm{PANI} /$ GNRs ternary composites were obtained by filtration and washed with deionized water and ethanol in sequence and dried at $60{ }^{\circ} \mathrm{C}$ under vacuum.

\subsection{Instruments and characterization}

The morphologies of the products were investigated by a scanning electron microscope (SEM, JSM-7800F JEOL Co., Japan) and a transmission electron microscope (TEM, JEM-2100 JEOL Co., Japan), respectively. Infrared spectra were recorded on a Varian 3100 FT-IR spectrometer by using pressed KBr pellets. X-ray diffraction (XRD) patterns of the composites were measured on a XRD, $\mathrm{Cu} \mathrm{K} \alpha$ radiation, Bruker D8 Advance, Germany. X-ray photoelectron spectroscopy (XPS) analysis was carried out on an ECSALAN 250 electron spectrometer using a monochromatized Al KR X-ray source (1486.71 eV photons).

2.5.1 Electrochemical measurement. The electrochemical properties of the composite were investigated on a CHI660E electrochemical workstation (Shanghai, China) with conventional three-electrode system at room temperature. To prepare the working electrodes, $80 \mathrm{wt} \%$ of active materials, $10 \mathrm{wt} \%$ acetylene black and $10 \mathrm{wt} \%$ PVDF were mixed to form 
a homogenous slurry. Then the slurry was pressed onto nickel foam current collectors with the surface area around $1 \mathrm{~cm}^{2}$. Finally, the nickel foam was dried at $70{ }^{\circ} \mathrm{C}$ for $8 \mathrm{~h}$ under vacuum. Electrochemical properties of the samples were investigated via cyclicvoltammetry (CV), galvanostatic chargedischarge (GCD) and electrochemical impedance spectroscopy (EIS) measurements. And the platinum electrode and saturated calomel electrode was used as counter and reference electrodes, respectively. And $1 \mathrm{M} \mathrm{Na}_{2} \mathrm{SO}_{4}$ solution was used as electrolyte. The potential range for $\mathrm{CV}$ tests was $0-0.8 \mathrm{~V}$ and the scan rate was $10-100 \mathrm{mV} \mathrm{s}^{-1}$. The potential range for charge-discharge tests was $0-0.8 \mathrm{~V}$. EIS measurements were carried out in the frequency range of $10^{5}$ to $0.01 \mathrm{~Hz}$ at $5 \mathrm{mV}$ amplitude referring to open circuit potential. Galvanostatic charge-discharge tests were conducted on a LAND CT2001A program testing system.

\section{Results and discussion}

As shown Fig. 1, $\mathrm{MnO}_{2} / \mathrm{PANI} / \mathrm{GNRs}$ composites were fabricated by two-step polymerization. By step-oxidation, there are lots of oxygen-containing groups, such as epoxy, hydroxyl and carboxyl groups in the structure of GNRs. ${ }^{26}$ These functional groups, acting as anchor sites, enable the aniline units subsequent in situ formation of PANI attaching on the surfaces and edges of GNRs. ${ }^{24}$ After first step polymerization, since the $\mathrm{MnO}_{2}$ nanorods can be acted as oxidant reactive template for oxidative polymerization of aniline, the aniline monomers absorbed on the surfaces of GNRs can be oxidized to PANI polymer around the surfaces of the $\mathrm{MnO}_{2}$ nanorods, accompanied by the

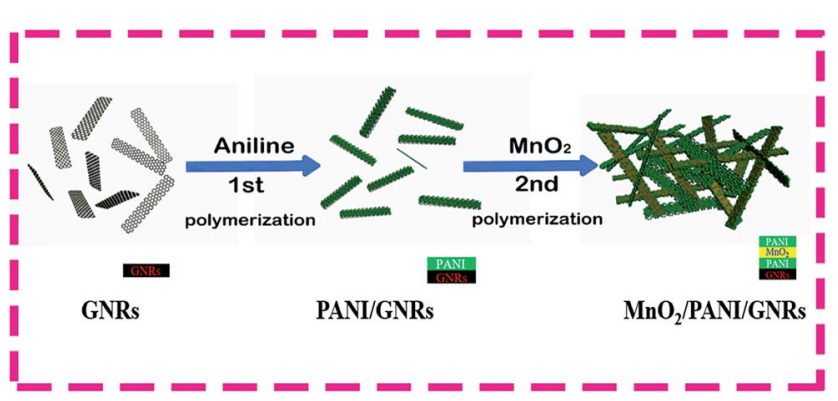

Fig. 1 Schematic illustration of fabrication of $\mathrm{MnO}_{2} / \mathrm{PANI} / \mathrm{GNRs}$. reduction of $\mathrm{MnO}_{2}$ to soluble $\mathrm{Mn}^{2+}$ ions. Therefore, the $\mathrm{MnO}_{2} /$ GNRs/PANI ternary composites with $\mathrm{MnO}_{2}$ and GNRs dispersed in the PANI matrix were obtained after second-step polymerization.

FT-IR spectra were first used to characterize the formation of $\mathrm{MnO}_{2} / \mathrm{PANI} / \mathrm{GNRs}$ composites. From Fig. 2a, regarding FT-IR spectrum of GNRs, there are characteristic peaks located at 1721,1238 and $1040 \mathrm{~cm}^{-1}$ which are attributed to the vibration of carbonyl, epoxy and hydroxyl groups, respectively. The peak of $1610 \mathrm{~cm}^{-1}$ is assigned to the stretching vibration of $\mathrm{C}=\mathrm{C}$ group. ${ }^{24,26}$ Towards to PANI, the absorption peaks located at 1570 and $1492 \mathrm{~cm}^{-1}$ are ascribed to the $\mathrm{C}=\mathrm{C}$ stretching deformation of the quinoid ring and benzenoid rings in the emeraldine salt. The peaks corresponding to the $\mathrm{C}-\mathrm{N}$ stretching of secondary aromatic amine and $\mathrm{C}=\mathrm{N}$ stretching appear at 1302 and $1152 \mathrm{~cm}^{-1}$, respectively. ${ }^{33-35}$ It is worth noting that the FTIR spectra of $\mathrm{MnO}_{2} / \mathrm{PANI} / \mathrm{GNRs}$ almost shows similar peaks as PANI, which indicates the PANI were successfully introduced into the $\mathrm{MnO}_{2} / \mathrm{PANI} / \mathrm{GNRs}$.

The XRD patterns were also demonstrated the formation of $\mathrm{MnO}_{2} / \mathrm{PANI} / \mathrm{GNRs}$. For $\mathrm{MnO}_{2}$ nanorods, significant XRD peaks were recorded at $2 \theta=12.98^{\circ}, 18.24^{\circ}, 25.72^{\circ}, 28.86^{\circ}, 37.66^{\circ}$, $41.90^{\circ}, 49.80^{\circ}, 56.28^{\circ}, 60.32^{\circ}, 65.60^{\circ}$ and $69.24^{\circ}$, which could be well-assigned to the (100), (200), (220), (310), (211), (301), (411), (600), (521), (002) and (541) crystal planes of a pure tetragonal the $\alpha-\mathrm{MnO}_{2}$ phase. ${ }^{32,36}$ For pure polymer of PANI, the diffraction peaks were located at $2 \theta=8.62^{\circ}, 16.34^{\circ}, 20.41^{\circ}, 25.32^{\circ}$ and $26.92^{\circ}$, illustrating that the in situ polymerized PANI are in highly doped emeraldine salt form. ${ }^{33,34}$ As for $\mathrm{MnO}_{2} / \mathrm{PANI} /$ GNRs, a board peak is centered at $2 \theta=26.92^{\circ}$, showing similar peaks as PANI except for that the characteristic peaks are becoming obscure, which reveals that the degree of crystallinity of PANI in $\mathrm{MnO}_{2} / \mathrm{PANI} / \mathrm{GNRs}$ become weak owing to the addition of GNRs and $\mathrm{MnO}_{2}$ nanorods. Moreover, no obvious diffraction peaks of GNRs were observed in $\mathrm{MnO}_{2} / \mathrm{PANI} / \mathrm{GNRS}$ indicating that the regular stacks of GNRs are separated by the incorporated $\mathrm{MnO}_{2}$ and PANI, which is beneficial to its final electrochemical property.

Further, SEM and TEM images provided the structure and morphologies of $\mathrm{MnO}_{2} / \mathrm{PANI} / \mathrm{GNRs}$. As shown in Fig. 3a, there is no tubulous structure and internal and external walls for GNRs, instead, ribbon-like thin graphene layer with crimped edges.
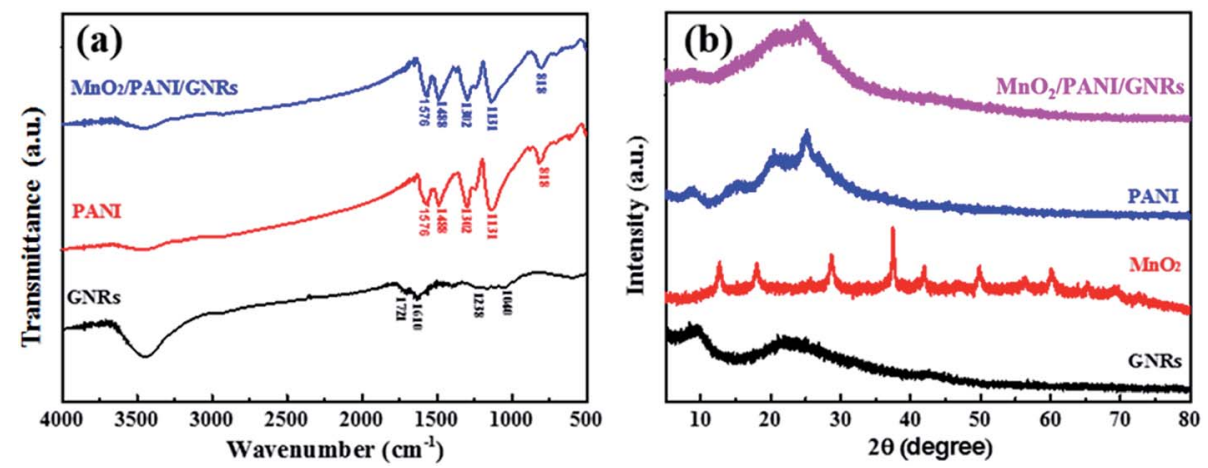

Fig. 2 (a) FT-IR spectra of GNRs, PANI and $\mathrm{MnO}_{2} / \mathrm{PANI} / \mathrm{GNR}$, (b) XRD of GNRs, $\mathrm{MnO}_{2}$, PANI and $\mathrm{MnO}_{2} / \mathrm{PANI} / \mathrm{GNR}$. 

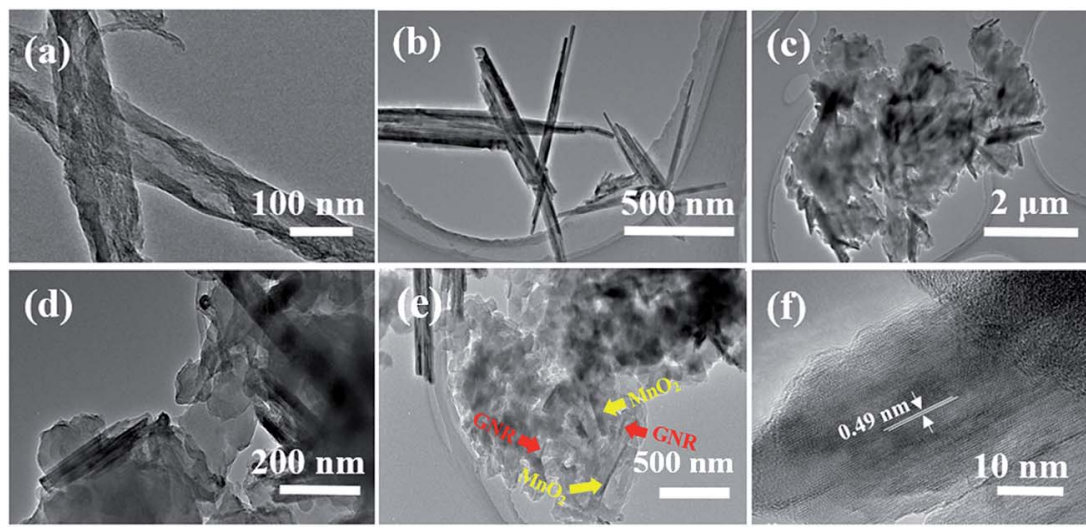

Fig. 3 TEM images of (a) GNRs, (b) $\mathrm{MnO}_{2}$ nanorods, (c-e) $\mathrm{MnO}_{2} /$ PANI/GNRs composites, (f) HRTEM image of $\mathrm{MnO}_{2} /$ PANI/GNRs composites.

From Fig. 3b, the as-prepared $\mathrm{MnO}_{2}$ nanoparticles exhibit rodlike structure. The mean width and length for the $\mathrm{MnO}_{2}$ nanorods are $103.74 \mathrm{~nm}$ and $1.95 \mu \mathrm{m}$, respectively (Fig. S1 and $\mathrm{S} 2$, ESI $\dagger$ ). After combination of PANI with two-step polymerization, the GNRs and $\mathrm{MnO}_{2}$ nanorods were filled into the PANI matrix. From Fig. 3c-e, there are distinct $\mathrm{MnO}_{2}$ nanorods embed in PANI. Due to the ultra-thin ribbon-like structure of GNRs, there is almost no GNRs presented in the SEM images of $\mathrm{MnO}_{2} / \mathrm{PANI} / \mathrm{GNRs}$ composites. As pointed in Fig. 3e, there are some curved GNRs inserted into the PANI matrix. As shown in Fig. 3f, the $\mathrm{MnO}_{2}$ nanorods exhibits well crystallized with interplanar distance is $0.49 \mathrm{~nm}$, corresponding to the (200) crystal plane. Brunauer-Emmett-Teller (BET) measurement was also conducted to characterize the structure of $\mathrm{MnO}_{2} / \mathrm{PANI} /$ GNRs ternary composite. As shown in Fig. S3a (ESI $\dagger$ ), the nitrogen adsorption-desorption isotherms of $\mathrm{MnO}_{2} / \mathrm{GNRs} /$ PANI ternary composite showed a typical type IV with a hysteresis loop at relative pressure $\left(P / P_{0}\right)$ of $0.4-1$, demonstrating the presence of porous structure of $\mathrm{MnO}_{2} / \mathrm{GNRs} / \mathrm{PANI}$ ternary composite. The Brunauer-Emmett-Teller (BET) specific surface area of the NDPC was estimated as $326.7 \mathrm{~m}^{2} \mathrm{~g}^{-1}$. The pore size distribution curve of $\mathrm{MnO}_{2} / \mathrm{GNRs} / \mathrm{PANI}$ ternary composite estimated by Barrett-Joyner-Halenda $(\mathrm{BJH})$, is illustrated in Fig. S3b (ESI $\dagger$ ). The $\mathrm{MnO}_{2} / \mathrm{GNRs} / \mathrm{PANI}$ ternary composite is meso-pores with an intensive pore size distribution of $\sim 4.07 \mathrm{~nm}$. The three-dimensional porous $\mathrm{MnO}_{2} / \mathrm{GNRs} / \mathrm{PANI}$ ternary composite with high specific surface can accommodate the volume change and facilitate penetration of electrolyte and fast diffusion of ions. ${ }^{37-40}$

Obviously, as illustrated in Fig. 4a, utilizing the interaction between the GNRs and aniline monomers, the GNRs were first wrapped with PANI polymer chains after first polymerization. Afterward, due to the introduction of $\mathrm{MnO}_{2}$ nanorods, the structures of composites were turned out to be a $3 \mathrm{D}$ porous structure with the GNRs interconnected with $\mathrm{MnO}_{2}$, together embedding into PANI matrix, which we can believe this unique structure provides an efficient path for electron and ion transport. ${ }^{35}$ As observed in Fig. $4 \mathrm{~b}-\mathrm{d}$, the $\mathrm{MnO}_{2} / \mathrm{PANI} /$ GNRs show the loose and porous 3D structure with embedding with $\mathrm{MnO}_{2}$ nanorods and GNRs. The elements mapping images in Fig. 4e$\mathrm{h}$ shows the uniform distributions of $\mathrm{MnO}_{2}$ and GNRs in PANI matrix.

To further understanding the composition of $\mathrm{MnO}_{2} / \mathrm{PANI} /$ GNRs, XPS was carried out and the results are shown in Fig. 5. As for GNRs, the carbon and oxygen signals were observed at 284.6 and $531.9 \mathrm{eV} \cdot{ }^{24}$ Obviously, after combination, the existences of $\mathrm{C}, \mathrm{N}, \mathrm{O}, \mathrm{Mn}$ elements in $\mathrm{MnO}_{2} / \mathrm{PANI} / \mathrm{GNRs}$ can be confirmed from the wide range as shown in Fig. 5b. The XPS spectra of $\mathrm{N}$ 1s can be decomposed into three distinct curves, related to $398.6 \mathrm{eV}(-\mathrm{N}=), 399.7 \mathrm{eV}(-\mathrm{NH}-)$ and $400.1 \mathrm{eV}\left(-\mathrm{N}^{+}-\right)$. More specifically, the peak located at $400.1 \mathrm{eV}$ is assigned to the quinoid amine and nitrogen cationic radical $(-\mathrm{N}=)$ and the one
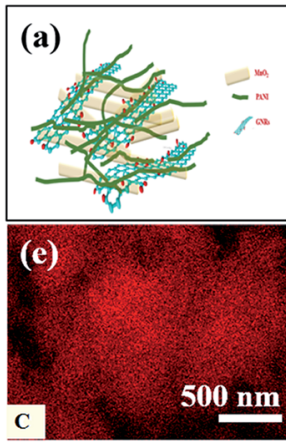
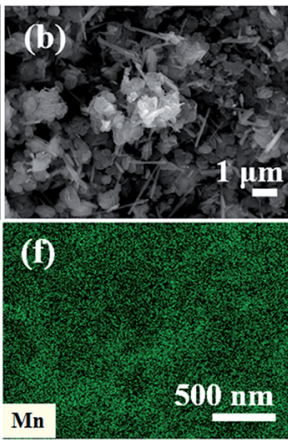
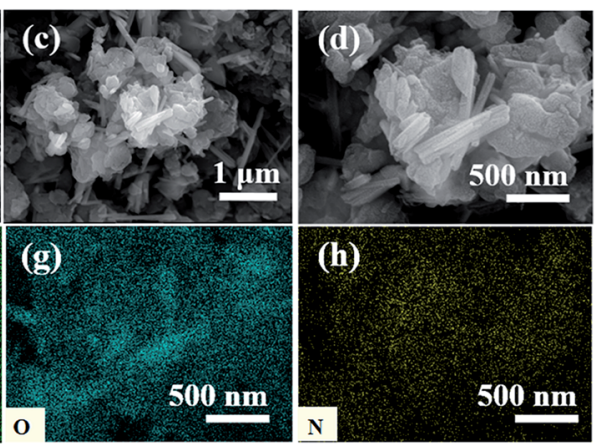

Fig. 4 (a) Structure schematic drawing for $\mathrm{MnO}_{2} / \mathrm{PANI} / \mathrm{GNRs}$ composites, (b and c) SEM images of $\mathrm{MnO}_{2} / \mathrm{PANI} / \mathrm{GNRs}$ composites with different magnifications, $(\mathrm{d}-\mathrm{h})$ elements mapping for $\mathrm{C}, \mathrm{Mn}, \mathrm{O}$ and $\mathrm{N}$ elements of $\mathrm{MnO}_{2} / \mathrm{PANI} / \mathrm{GNRs}$ composites. 

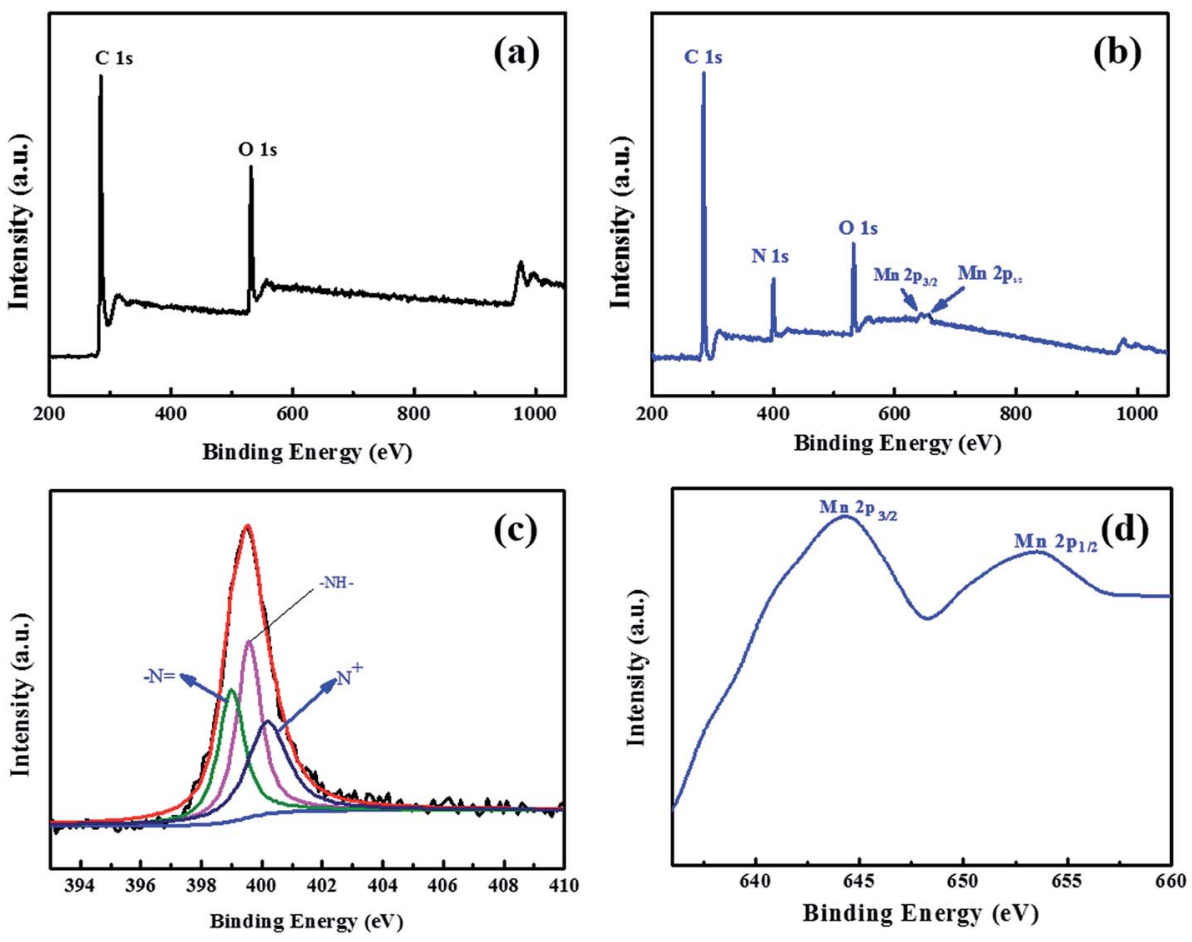

Fig. 5 XPS surveys of (a) GNRs and (b) $\mathrm{MnO}_{2} /$ PANI/GNRs, high-resolution N 1s (c) and Mn 2p (d) core-level XPS spectra of MnO 2 /PANI/GNRs.

at $398.6 \mathrm{eV}(-\mathrm{N}=)$ is due to benzenoid amine. ${ }^{35}$ The Mn $2 \mathrm{p}$ XPS spectrum exhibits two characteristic peaks, $\mathrm{Mn} 2 \mathrm{p}_{3 / 2}$ at $643.2 \mathrm{eV}$ and $\mathrm{Mn} 2 \mathrm{p}_{1 / 2}$ peak at $654.8 \mathrm{eV}$, which are attributed to the spinorbit peaks of $\alpha-\mathrm{MnO}_{2}$. The peak-to-peak separation between $\mathrm{Mn} 2 \mathrm{p}_{1 / 2}$ and $\mathrm{Mn} 2 \mathrm{p}_{3 / 2}$ level is $11.6 \mathrm{eV}$, which is approximately the same value as that literature for $\mathrm{MnO}_{2},{ }^{28,32,35}$ confirming the presence of $\mathrm{MnO}_{2}$ in composites. From the XPS data of $\mathrm{MnO}_{2} /$ PANI/GNRs composite, the atomic ratios of $\mathrm{C}, \mathrm{N}, \mathrm{O}, \mathrm{Mn}$ elements are $68.86 \%, 8.31 \%, 10.51 \%$ and $12.32 \%$, respectively.

The wrapped PANI with $\mathrm{MnO}_{2}$ and GNRs can not only offer significant capacitance because of it pseudocapacitance character but can also enhance the contact between GNRs and $\mathrm{MnO}_{2}$ nanorods, thus, improving the capacitive performance. For understanding the electrochemical property and qualify the specific capacitance of the $\mathrm{MnO}_{2} / \mathrm{PANI} / \mathrm{GNR}$ composites, the cyclic voltammograms (CV) measurements of electrode materials for $\mathrm{MnO}_{2}, \mathrm{MnO}_{2} / \mathrm{GNRs}$ and $\mathrm{MnO}_{2} / \mathrm{PANI} / \mathrm{GNRs}$ composites were performed in a $1 \mathrm{M} \mathrm{Na}_{2} \mathrm{SO}_{4}$ aqueous solution using a threeelectrode system. As shown in Fig. 6a, under the electrochemical window from 0 to $0.8 \mathrm{~V}$ with a low scan rate of $5 \mathrm{mV}$ $\mathrm{s}^{-1}$, the $\mathrm{MnO}_{2}$ electrode exhibits rectangular and symmetric CV curves, indicating the ideal pseudocapacitance feature of $\mathrm{MnO}_{2}$. Incorporating with GNR, the increased area of $\mathrm{CV}$ curves is probably due to the combined electric double-layer and pseudocapacitive contribution to the total capacitance. ${ }^{25}$ After introduction of PANI, the $\mathrm{CV}$ curve for $\mathrm{MnO}_{2} / \mathrm{PANI} / \mathrm{GNRS}$ ternary composites is different from that of $\mathrm{MnO}_{2}$ and $\mathrm{MnO}_{2} /$ GNRs. There are two pairs redox peaks appeared for $\mathrm{MnO}_{2} /$ PANI/GNRs. It is attributed to the leucoemeraldine-emeraldine transition and the emeraldine-pernigraniline transition. Consequently, the capacitance of $\mathrm{MnO}_{2} / \mathrm{PANI} / \mathrm{GNRs}$ composites partially comes from faradaic reactions of PANI at the electrode/ electrolyte surface, which is different from that of the electric double-layer capacitance of GNRs and pseudocapacitance of $\mathrm{MnO}_{2} \cdot{ }^{35,36}$ Fig. $6 \mathrm{c}$ shows the $\mathrm{CV}$ curves of $\mathrm{MnO}_{2} / \mathrm{PANI} / \mathrm{GNRs}$ at different scan rates. The current density of the $\mathrm{MnO}_{2} / \mathrm{PANI} /$ GNRs composites increased with the scan rate and the curve shape is stable. The results demonstrated that the $\mathrm{MnO}_{2} / \mathrm{PANI} /$ GNRs has good electrochemical stability. Galvanostatic charge/ discharge plots $\left(1 \mathrm{Ag}^{-1}\right)$ of $\mathrm{MnO}_{2}, \mathrm{MnO}_{2} / \mathrm{GNRs}$ and $\mathrm{MnO}_{2} / \mathrm{PANI} /$ GNRs composites were presented in Fig. 6b. During the charging and discharging steps, all the curves exhibit an equilateral triangle shape, indicating high reversibility of the hybrid materials during charge/discharge process. The specific capacitance can be calculated according to $C_{\mathrm{m}}=I \times \Delta t /(\Delta V \times m)$ from the discharge curves, where $C_{\mathrm{m}}$ is the specific capacitance $\left(\mathrm{F}^{-1}\right), I$ is the constant discharge current (A), $\Delta t$ is the discharge time (s), $\Delta V$ is the potential window (V), $m$ is the mass of active material in the working electrode $(\mathrm{g}) .^{32,35}$ The gravimetric specific capacitances of $\mathrm{MnO}_{2}, \mathrm{GNRs} / \mathrm{MnO}_{2}$ and $\mathrm{MnO}_{2}$ / PANI/GNRs were 136, 245 and $472 \mathrm{~F} \mathrm{~g}^{-1}$ at the current density of $1 \mathrm{~A} \mathrm{~g}^{-1}$, respectively. Obviously, the specific capacitance of $\mathrm{MnO}_{2} / \mathrm{PANI} / \mathrm{GNRs}$ ternary composite is higher than that of $\mathrm{MnO}_{2}$ and $\mathrm{GNRs} / \mathrm{MnO}_{2}$, indicating the synergistic effect among GNRs, $\mathrm{MnO}_{2}$ and PANI. From Fig. 6d, the specific capacitance for $\mathrm{MnO}_{2} / \mathrm{PANI} / \mathrm{GNRs}$ ternary composite at 1, 2, 5 and $10 \mathrm{~A} \mathrm{~g}^{-1}$ were $472,385,347$ and $306 \mathrm{~F} \mathrm{~g}^{-1}$. From Table 1, the specific capacitance for $\mathrm{MnO}_{2} / \mathrm{PANI} / \mathrm{GNRs}$ ternary composite was higher than those of other reported previous reported $\mathrm{GNR}-\mathrm{MnO}_{2}$ $(18 \%),{ }^{41} \mathrm{GNR} / \mathrm{MnO}_{2},{ }^{42} \mathrm{PANI}-\mathrm{MnO}_{2}$ nanowires, ${ }^{43} \mathrm{PANI} / \mathrm{MnO}_{2},{ }^{44}$ and PANI-GNRs-40. ${ }^{45}$ From Fig. 7a, generally, the specific capacitance decreases with the increase of current density 

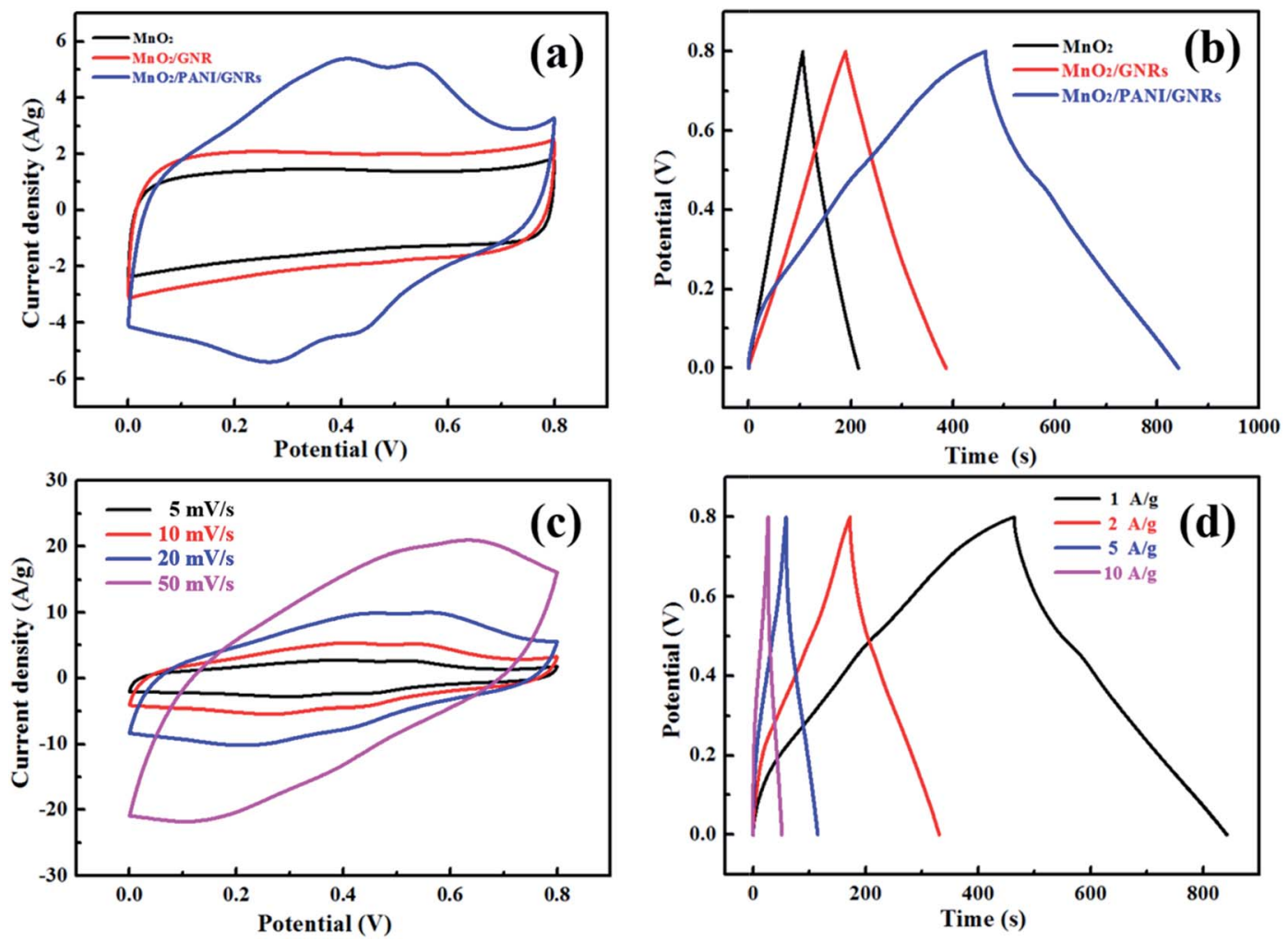

Fig. 6 (a) $\mathrm{CV}$ curves of $\mathrm{MnO}_{2}$, GNRs and $\mathrm{MnO}_{2} / \mathrm{PANI} / \mathrm{GNRs}$ composites at the scan rate of $5 \mathrm{mV} \mathrm{s}^{-1}$, (b) galvanostatic charge/discharge plots of $\mathrm{MnO}_{2}, \mathrm{MnO}_{2} / \mathrm{GNRs}$ and $\mathrm{MnO}_{2} / \mathrm{PANI} / \mathrm{GNRs}$ composites at a current density of $1 \mathrm{~A} \mathrm{~g}^{-1}$. (c) CV curves of MnO $/$ /PANI/GNRs composites at different scan rates and (d) galvanostatic charge/discharge plots of $\mathrm{MnO}_{2} / \mathrm{PANI} / \mathrm{GNRs}$ composites at different current densities.

Table 1 Comparison of electrochemical performance of the other reported composites involving $\mathrm{MnO}_{2}, \mathrm{GNRs}$ PANI combinations (binary or ternary) ${ }^{a}$

\begin{tabular}{|c|c|c|c|c|c|}
\hline Materials & $C\left(\mathrm{~F} \mathrm{~g}^{-1}\right)$ & EI & $R$ & $\mathrm{~T}$ & Ref \\
\hline $\mathrm{GNR}^{-\mathrm{MnO}_{2}}(18 \%)$ & 305 & $\mathrm{PAAK} / \mathrm{KCl}$ & $0.5 \mathrm{~A} \mathrm{~g}^{-1}$ & 2-electrode GCD & 41 \\
\hline $\mathrm{GNR} / \mathrm{MnO}_{2}$ & 266 & $1 \mathrm{M} \mathrm{Na}_{2} \mathrm{SO}_{4}$ & $1 \mathrm{Ag}^{-1}$ & 3-electrode GCD & 42 \\
\hline $\mathrm{PANI} / \mathrm{MnO}_{2}$ & 320 & $1 \mathrm{M} \mathrm{Na}_{2} \mathrm{SO}_{4}$ & $1 \mathrm{~A} \mathrm{~g}^{-1}$ & 3-electrode GCD & 44 \\
\hline PANI-GNRs-40 & 340 & $1 \mathrm{M} \mathrm{H}_{2} \mathrm{SO}_{4}$ & $0.25 \mathrm{~A} \mathrm{~g}^{-1}$ & 3-electrode GCD & 45 \\
\hline CGNR/PANI/ $\mathrm{MnO}_{2}$ & 496 & $1 \mathrm{M} \mathrm{H}_{2} \mathrm{SO}_{4}$ & $1 \mathrm{Ag}^{-1}$ & 3-electrode GCD & 46 \\
\hline
\end{tabular}

${ }^{a}$ El: electrolyte type; $R$ : current density; T: testing method.

because the diffusion of ions from the electrolyte can gain access to almost all available area of the electrode at low current density, therefore, reaching a higher capacitance. As the current density increases, the capacitance decreases because of the effective interaction between the ion and the electrode is greatly reduced and the deep inside the material does not actually contribute to the capacitance. ${ }^{32}$ The specific capacitance of $\mathrm{MnO}_{2} / \mathrm{PANI} / \mathrm{GNRs}$ is $472 \mathrm{~F} \mathrm{~g}^{-1}$ at $1 \mathrm{~A} \mathrm{~g}^{-1}$ and as high as $306 \mathrm{~F}$ $\mathrm{g}^{-1}$ even at $10 \mathrm{~A} \mathrm{~g}^{-1}$, with a capacitance retention of $65 \%$. While the specific capacitance of $\mathrm{MnO}_{2}$ and $\mathrm{MnO}_{2} / \mathrm{GNRs}$ reaches $136 \mathrm{~F}$ $\mathrm{g}^{-1}$ and $245 \mathrm{~F} \mathrm{~g}^{-1}$ at a current density of $1 \mathrm{~A} \mathrm{~g}^{-1}$ and remains at $75 \mathrm{~F} \mathrm{~g}^{-1}$ and $163 \mathrm{~F} \mathrm{~g}^{-1}$ at $10 \mathrm{~A} \mathrm{~g}^{-1}$ with a capacitance retention of $55 \%$ and $63 \%$, respectively. The $\mathrm{MnO}_{2} / \mathrm{PANI} / \mathrm{GNRs}$ hybrids not only exhibit high capacitance but also high stable electrochemical performance at high current densities. This may because the special composite structure of $\mathrm{MnO}_{2} / \mathrm{PANI} / \mathrm{GNRs}$ provides numerous porous which can effectively facilitate the ions access to the inside of the composites. ${ }^{14,17,35}$ On the other hand, the synergist effect between GNRs, $\mathrm{MnO}_{2}$ and PANI significantly reduce the interval conductivity of the composites. As a result, the $\mathrm{MnO}_{2} / \mathrm{PANI} / \mathrm{GNRs}$ composites exhibit high capacity retention at a high current density.

Due to the synergistic effect of GNRs, $\mathrm{MnO}_{2}$ and PANI, $\mathrm{MnO}_{2} / \mathrm{PANI} / \mathrm{GNRs}$ also exhibits good cyclic electrochemical performance. As shown in Fig. 7b, the galvanostatic chargingdischarging cycles for $\mathrm{MnO}_{2}, \mathrm{MnO}_{2} / \mathrm{GNRs}$ and $\mathrm{MnO}_{2} / \mathrm{PANI} /$ 

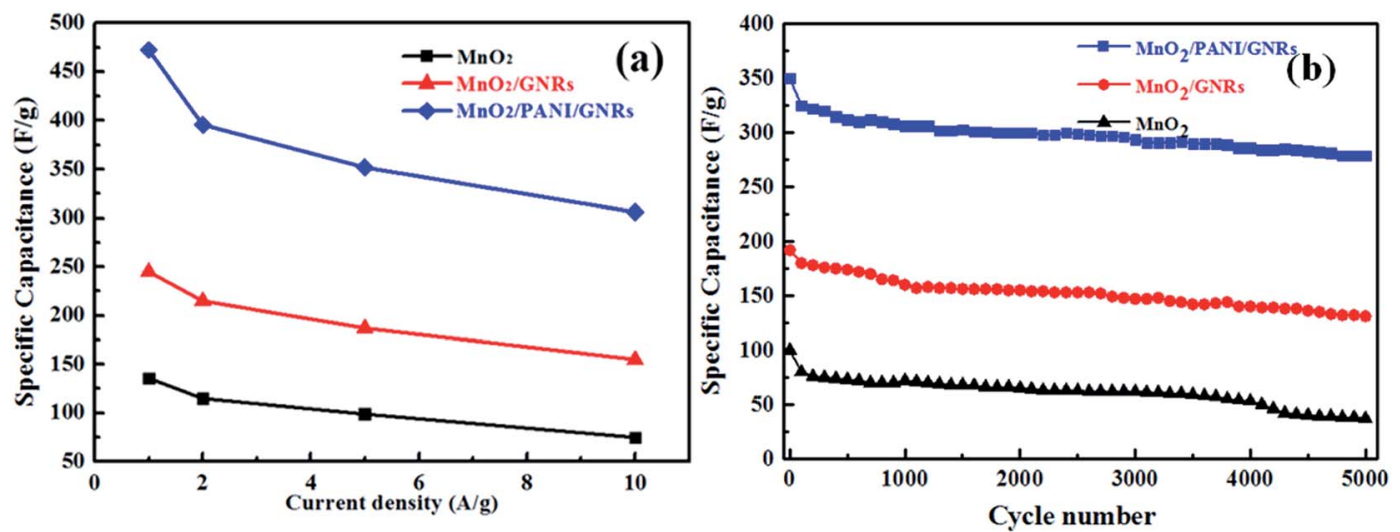

Fig. 7 (a) Specific capacitance versus current density for GNRs, $\mathrm{MnO}_{2}$ and PANI, $\mathrm{MnO}_{2} / \mathrm{PANI} / \mathrm{GNRs}$ composites and (b) charge-discharge cycling performances for GNRs, $\mathrm{MnO}_{2}$ and PANI, $\mathrm{MnO}_{2}$ /PANI/GNRs composites at a current density of $10 \mathrm{Ag}^{-1}$.
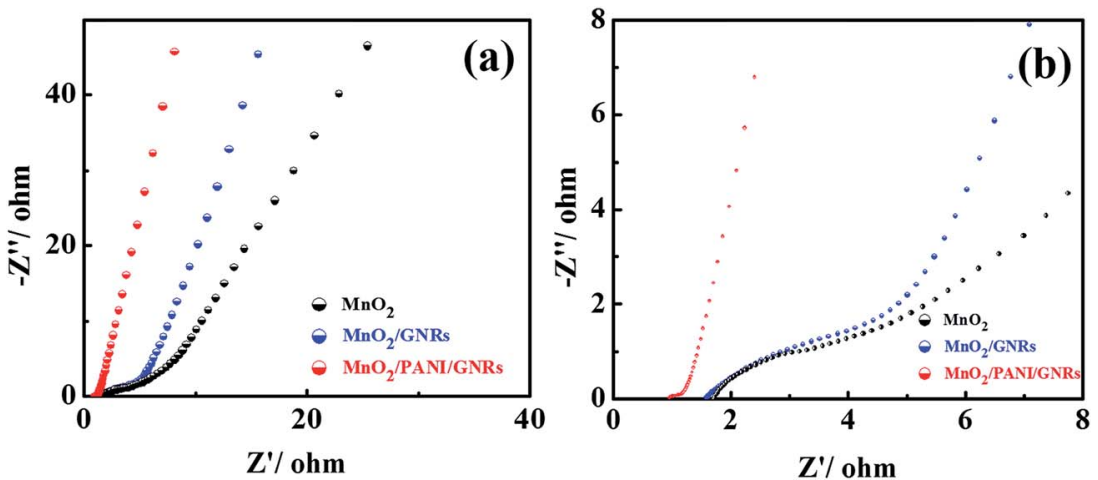

Fig. 8 (a) Nyquist plots of $\mathrm{MnO}_{2}, \mathrm{MnO}_{2} / \mathrm{GNRs}$ and $\mathrm{MnO}_{2} / \mathrm{PANI} / \mathrm{GNRs}$ ternary composites and (b) partial enlarged $\mathrm{Nyquist} \mathrm{plots} \mathrm{of} \mathrm{MnO}_{2}$, $\mathrm{MnO}_{2} /$ GNRs and $\mathrm{MnO}_{2} /$ PANI/GNRs.

GNRs were carried out at a current density of $10 \mathrm{~A} \mathrm{~g}^{-1}$ within a voltage range from 0.0 to $0.8 \mathrm{~V}$ for 5000 cycles. After 5000 cycles, the $\mathrm{MnO}_{2}$ and $\mathrm{MnO}_{2} / \mathrm{GNRs}$-based electrode performed only $35.5 \%$ and $68.4 \%$ capacitance retention after 1000 cycles. But for $\mathrm{MnO}_{2} / \mathrm{PANI} / \mathrm{GNRs}$, there is a minor degradation of the specific capacitance with $79.7 \%$ of the initial capacitance preserved after 5000 charge-discharge cycles. The improved cycle stability may be deprived from the outstanding stability of the 3D structure constituted by $\mathrm{MnO}_{2}$ nanorods and GNRs, which exhibits excellent mechanical properties. And it effectively prevents the swelling and shrinking of $\mathrm{MnO}_{2} / \mathrm{PANI} / \mathrm{GNRs}$ nanostructure during the long-term cycle test. In addition, the 3D porous structure can greatly reduce the diffusion and migration length of the electrolyte ions during the fast charge/ discharge process, and increases the electrochemical utilization of all the active sites. ${ }^{47}$

To further understand the improvement of electrochemical performance of the $\mathrm{MnO}_{2} / \mathrm{PANI} / \mathrm{GNRs}$ ternary composite electrode, electrochemical impedance spectroscopy (EIS) was carried out within a frequency range from $100 \mathrm{kHz}$ down to $0.01 \mathrm{~Hz}$. The observed semicircle corresponds to a higherfrequency region, whereas the inclined straight line corresponds to the low-frequency. The depression in the semicircle is modeled as the parallel combination of an interfacial charge transfer resistance and double-layer capacitance. ${ }^{48}$ The inclined straight line at low-frequency represents the ion diffusion and transport due to the pseudocapacitance. ${ }^{49}$ Fig. 8 represents the Nyquist plots for $\mathrm{MnO}_{2}, \mathrm{MnO}_{2} / \mathrm{GNRs}$ and $\mathrm{MnO}_{2} / \mathrm{PANI} / \mathrm{GNRs}$ electrodes. It is known that the charge transfers resistance $\left(R_{\mathrm{ct}}\right)$ can be directly measured by the semicircular diameter. ${ }^{11}$ It can be observed that $\mathrm{MnO}_{2} / \mathrm{PANI} /$ GNRs exhibits a smaller arc diameter than $\mathrm{MnO}_{2}$ and $\mathrm{MnO}_{2} /$ GNRs, indicating that $\mathrm{MnO}_{2} / \mathrm{PANI} / \mathrm{GNRs}$ has low charge transfers resistance with faster ion diffusion rate in $\mathrm{Na}_{2} \mathrm{SO}_{4}$ solution. Compared to $\mathrm{MnO}_{2} / \mathrm{PANI} / \mathrm{GNRs}$, the $\mathrm{MnO}_{2} / \mathrm{GNRs}$ with a larger arc diameter exhibited slower diffusion rate due to the introduction of GNRs. While the $\mathrm{MnO}_{2}$ presents a larger arc diameter, which might be attributed to the poor conductivity of $\mathrm{MnO}_{2}$. The lowest charge transfer resistance in ternary $\mathrm{MnO}_{2} / \mathrm{PANI} / \mathrm{GNRs}$ implied the excellent rate capability. In addition, in the low frequency region, the slope of the curve shows the Warburg impedance, representing the diffusion and transport of ions in the electrode. ${ }^{47} \mathrm{MnO}_{2} / \mathrm{PANI} /$ GNRs shows the most vertical line at lower frequencies, further indicating its high capacitive behavior. 


\section{Conclusion}

In summary, unique $\mathrm{MnO}_{2} / \mathrm{PANI} / \mathrm{GNRs}$ composite have been successfully synthesized via a two-step in situ process. The asprepared composites electrode exhibits an excellent specific capacitance of $472 \mathrm{~F} \mathrm{~g}^{-1}$ at the current density of $1 \mathrm{~A} \mathrm{~g}^{-1}$ in $1 \mathrm{M}$ $\mathrm{Na}_{2} \mathrm{SO}_{4}$ aqueous solution. In addition, the $\mathrm{MnO}_{2} / \mathrm{PANI} / \mathrm{GNRS}$ show high electrochemical stability in cycling stability with $79.7 \%$ capacitance retention after 5000 cycles. Such intriguing electrochemical performance was because of the unique 3D porous structure constituted by $\mathrm{MnO}_{2}$ and GNRs, leading to an excellent rate performance and cycling stability. On the other hand, the synergistic effects of GNRs, PANI and $\mathrm{MnO}_{2}$ have overcome the disadvantages of each component to some extent, therefore, resulting in a robust capacitive performance. The facile and effective synthetic strategy, we believe, can not only be employed for the fabrication of robust high-performance energy storage material containing carbon materials, metal oxide and conducting polymers, but also can be extended to other material preparation for the development of advanced electrode materials, which may pave an avenue for the practical applications of supercapacitors.

\section{Acknowledgements}

This research was supported by leading talent program of Shanghai, Sailing program of Shanghai Science and Technology Commission (15YF1404700), Startup Fund for New Talent, Shanghai University of Electric Power (K-2014-046), National Nature Science Foundation of China (No. 21604051) and Science and Technology Commission of Shanghai Municipality (No. 14JC1402500). The study was also sponsored by "Chenguang Program" supported by Shanghai Education Development Foundation and Shanghai Municipal Education Commission (14CG54). Additionally, the authors are greatly indebted to the financial supports from the Projects of Science and Technology Department of Guizhou Province (No. [2016] 7079), the Projects of Education Department of Guizhou Province ([2016]162). Finally, we thank the International Joint Laboratory on Resource Chemistry (IJLRC).

\section{References}

1 G. Wang, L. Zhang and J. Zhang, Chem. Soc. Rev., 2012, 41, 797-828.

2 M. Salanne, B. Rotenberg, K. Naoi, K. Kaneko, P. L. Taberna, C. P. Grey, B. Dunn and P. Simon, Nat. Energy, 2016, 1, 16070.

3 W. Chen, R. B. Rakhi, L. Hu, X. Xie, Y. Cui and

H. N. Alshareef, Nano Lett., 2011, 11, 5165-5172.

4 P. Simon and Y. Gogotsi, Nat. Mater., 2008, 7, 845-854.

5 B. Fang, A. Bonakdarpour, M.-S. Kim, J. H. Kim, D. P. Wilkinson and J.-S. Yu, Microporous Mesoporous Mater., 2013, 182, 1-7.

6 B. Fang, J. H. Kim, M.-S. Kim, A. Bonakdarpour, A. Lam, D. P. Wilkinson and J.-S. Yu, J. Mater. Chem., 2012, 22, 19031-19038.

7 B. Fang and L. Binder, J. Power Sources, 2006, 163, 616-622.
8 H. Ji, X. Zhao, Z. Qiao, J. Jung, Y. Zhu, Y. Lu, L. L. Zhang, A. H. MacDonald and R. S. Ruoff, Nat. Commun., 2014, 5, 3317.

9 F. Bonaccorso, L. Colombo, G. Yu, M. Stoller, V. Tozzini, A. C. Ferrari, R. S. Ruoff and V. Pellegrini, Science, 2015, 347, 1246501.

10 L. Q. Mai, A. Minhas-Khan, X. Tian, K. M. Hercule, Y. L. Zhao, X. Lin and X. Xu, Nat. Commun., 2013, 4, 2923.

11 Y. Liu, D. Yan, R. Zhuo, S. Li, Z. Wu, J. Wang, P. Ren, P. Yan and Z. Geng, J. Power Sources, 2013, 242, 78-85.

12 B. Brown, I. A. Cordova, C. B. Parker, B. R. Stoner and J. T. Glass, Chem. Mater., 2015, 27, 2430-2438.

13 C. Yuan, H. B. Wu, Y. Xie and X. W. Lou, Angew. Chem., 2014, 53, 1488-1504.

14 C. Pan, H. Gu and L. Dong, J. Power Sources, 2016, 303, 175181.

15 D. Wang, Y. Xiao, X. Luo, Z. Wu, Y.-J. Wang and B. Fang, ACS Sustainable Chem. Eng., 2017, 5, 2509-2515.

16 M. Kim, C. Lee and J. Jang, Adv. Funct. Mater., 2014, 24, 24892499.

17 H. Cao, X. Zhou, Y. Zhang, L. Chen and Z. Liu, J. Power Sources, 2013, 243, 715-720.

18 N. Ashok Kumar and J. B. Baek, Chem. Commun., 2014, 50, 6298-6308.

19 M. N. Hyder, R. Kavian, Z. Sultana, K. Saetia, P.-Y. Chen, S. W. Lee, Y. Shao-Horn and P. T. Hammond, Chem. Mater., 2014, 26, 5310-5318.

20 L. Wang, X. Lu, S. Lei and Y. Song, J. Mater. Chem. A, 2014, 2, 4491-4509.

21 Y. Huang, J. Liang and Y. Chen, Small, 2012, 8, 1805-1834.

22 D. V. Kosynkin, A. L. Higginbotham, A. Sinitskii, J. R. Lomeda, A. Dimiev, B. K. Price and J. M. Tour, Nature, 2009, 458, 872-876.

23 L. Jiao, L. Zhang, X. Wang, G. Diankov and H. Dai, Nature, 2009, 458, 877-880.

24 D. K. James and J. M. Tour, Macromol. Chem. Phys., 2012, 213, 1033-1050.

25 L. Li, A. R. Raji, H. Fei, Y. Yang, E. L. Samuel and J. M. Tour, ACS Appl. Mater. Interfaces, 2013, 5, 6622-6627.

26 F.-H. Hsu and T.-M. Wu, Synth. Met., 2014, 198, 188-195.

27 V. Sahu, S. Shekhar, R. K. Sharma and G. Singh, ACS Appl. Mater. Interfaces, 2015, 7, 3110-3116.

28 L. Li, A. R. Raji and J. M. Tour, Adv. Mater., 2013, 25, 62986302.

29 Q. Li, J. Liu, J. Zou, A. Chunder, Y. Chen and L. Zhai, J. Power Sources, 2011, 196, 565-572.

30 G. Wang, Q. Tang, H. Bao, X. Li and G. Wang, J. Power Sources, 2013, 241, 231-238.

31 P. Xiong, C. Hu, Y. Fan, W. Zhang, J. Zhu and X. Wang, J. Power Sources, 2014, 266, 384-392.

32 K. Dai, L. Lu, C. Liang, J. Dai, Q. Liu, Y. Zhang, G. Zhu and Z. Liu, Electrochim. Acta, 2014, 116, 111-117.

33 M. Kim, C. Lee, Y. D. Seo, S. Cho, J. Kim, G. Lee, Y. K. Kim and J. Jang, Chem. Mater., 2015, 27, 6238-6248.

34 Q. Hao, X. Xia, W. Lei, W. Wang and J. Qiu, Carbon, 2015, 81, 552-563. 
35 G. Han, Y. Liu, L. Zhang, E. Kan, S. Zhang, J. Tang and W. Tang, Sci. Rep., 2014, 4, 4824.

36 H.-M. Lee, G. H. Jeong, D. W. Kang, S.-W. Kim and C.-K. Kim, J. Power Sources, 2015, 281, 44-48.

37 B. Fang, Y. Xing, A. Bonakdarpour, S. Zhang and D. P. Wilkinson, ACS Sustainable Chem. Eng., 2015, 3, 2381-2388.

38 B. Fang, A. Bonakdarpour, K. Reilly, Y. Xing, F. Taghipour and D. P. Wilkinson, ACS Appl. Mater. Interfaces, 2014, 6, 15488-15498.

39 B. Fang, J. H. Kim, M.-S. Kim and J.-S. Yu, Acc. Chem. Res., 2013, 46, 1397-1406.

40 H. Xia, Y. Wang, J. Lin and L. Lu, Nanoscale Res. Lett., 2012, 7, 33.

41 M. Liu, W. W. Tjiu, J. Pan, C. Zhang, W. Gao and T. Liu, Nanoscale, 2014, 6, 4233-4242.
42 M.-S. Wu and Y.-H. Fu, Carbon, 2013, 60, 236-245.

43 L. Chen, Z. Song, G. Liu, J. Qiu, C. Yu, J. Qin, L. Ma, F. Tian and W. Liu, J. Phys. Chem. Solids, 2013, 74, 360-365.

44 M. Liu, Y.-E. Miao, C. Zhang, W. W. Tjiu, Z. Yang, H. Peng and T. Liu, Nanoscale, 2013, 5, 7312-7320.

45 L. Li, A.-R. O. Raji, H. Fei, Y. Yang, E. L. G. Samuel and J. M. Tour, ACS Appl. Mater. Interfaces, 2013, 5, 6622-6627.

46 Z. Yin, H. Zhou, C. Fu, N. Zhang, D. Liu and Y. Kuang, RSC Adv., 2016, 6, 41142-41150.

47 X. Sun, M. Gan, L. Ma, H. Wang, T. Zhou, S. Wang, W. Dai and H. Wang, Electrochim. Acta, 2015, 180, 977-982.

48 Y. Liu, Y. Ma, S. Guang, F. Ke and H. Xu, Carbon, 2015, 83, 79-89.

49 H. Li, Y. He, V. Pavlinek, Q. Cheng, P. Saha and C. Li, J. Mater. Chem. A, 2015, 3, 17165-17171. 